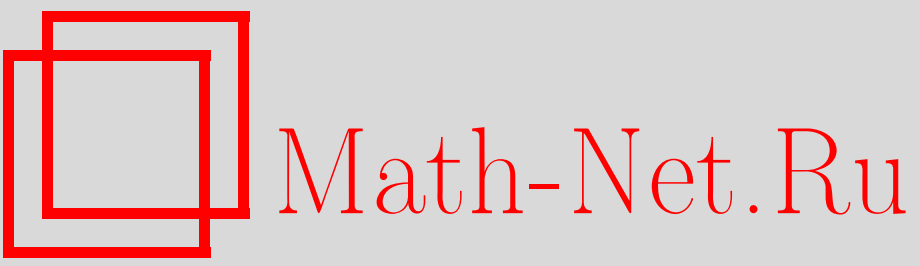

С. А. Апикян, М. А. Барсамян, К. Д. Эфтимиу, Геометрические свойства $W$ алгебр и модель Тоды, ТМФ, 2004, том 138, номер 2, 179-192

DOI: https://doi.org/10.4213/tmf16

Использование Общероссийского математического портала Math-Net.Ru подразумевает, что вы прочитали и согласны с пользовательским соглашением

http://www.mathnet.ru/rus/agreement

Параметры загрузки:

IP: 18.234 .156 .22

26 апреля 2023 г., 03:43:04 
ТЕОРЕТИЧЕСКАЯ

И МАТЕМАТИЧЕСКАЯ

ФИЗИКА

Том 138, № 2

февраль, 2004

(C) 2004 г. . А. Апикян*, М. А. Барсамян ${ }^{\dagger}$, К. Д. Эфтимиу

\title{
ГЕОМЕТРИЧЕСКИЕ СВОЙСТВА $W$-АЛГЕБР И МОДЕЛЬ ТОДЫ
}

\begin{abstract}
Строятся минимальные модели $W$-алгебр на гиперэллиптических римановых поверхностях. С помощью гипотезы Полякова функция распределения в теории поля Тоды на некоторой гиперэллиптической поверхности сводится к произведению функций распределения: теории "свободного поля" на сфере с включенными вершинными операторами Тоды и теории свободного скалярного поля с антипериодическими граничными условиями и включенными полями твистов.
\end{abstract}

Ключевые слова: конформная теория поля, интегрируемые системы, теория поля Тоды, гиперэллиптические поверхности.

\section{1. ВВЕДЕНИЕ}

В настояшее время квантовая конформная теория поля (КТП) на компактных римановых поверхностях актуальна по двум основным причинам. Во-первых, такая теория представляет интерес с математической точки зрения [1], во-вторых, она важна для понимания пертурбативного описания физики критических и некритических струн [2]. Однако аналитические вычисления на компактных римановых поверхностях возможны только в ограниченном количестве случаев. В настоящей работе мы даем описание $W$-алгебры и модели Тоды для некоторого подкласса римановых поверхностей - гиперэллиптических, имеюших то преимушество, что большинство вычислений на них можно проводить аналитически. Накопленньй опыт таких вычислений позволит нам сначала полнее изучить римановы $\mathbb{Z}_{n}$-поверхности, а затем и более общие случаи. Гиперэллиптические поверхности (ГЭП) широко используются в двухпетлевых вычислениях в теории струн и в теориях интегрируемых моделей [3] и Виттена-Зайберга [4], а также в областях, объединяюших эти теории [5].

\footnotetext{
* Ереванский государственный университет, Ереван, Армения. E-mail: apikyan@1x2.yerphi.am

${ }^{\dagger}$ Department of Mathematics, State University of New York, Stony Brook, New York, USA. E-mail: markbarsamian@yahoo.com

${ }^{\ddagger}$ Department of Physics, University of Central Florida, Orlando, Florida, USA. E-mail: costas@physics.ucf.edu
} 
Конформные преобразования в двумерной теории поля порождаются тензором энергии-импульса $T^{\mu \nu}$. Голоморфные (антиголоморфные) преобразования $z \rightarrow f(z)(\bar{z} \rightarrow$ $\bar{f}(\bar{z}))$ порождаются голоморфной (антиголоморфной) компонентой этого тензора $T(z)=$ $T^{11}-T^{22}+2 i T^{12}\left(\bar{T}(\bar{z})=T^{11}-T^{22}-2 i T^{12}\right)$. При бесконечно малых (инфинитезимальных ) конформных преобразованиях $z \rightarrow z+\epsilon(z)(\bar{z} \rightarrow \bar{z}+\bar{\epsilon}(\bar{z}))$ образующие, соответствующие параметрам $\epsilon_{n}$ в разложениях Лорана функции $\epsilon=\sum_{n=-\infty}^{\infty} \epsilon_{n} z^{n+1}$ $\left(\bar{\epsilon}=\sum_{n=-\infty}^{\infty} \bar{\epsilon}_{n} \bar{z}^{n+1}\right),-$ это операторы $L_{n}\left(\bar{L}_{n}\right)$, которые являются коэффициентами Лорана в разложении тензора энергии-импульса:

$$
T(z)=\sum_{n \in Z} L_{n} z^{-n-2} .
$$

Образующие конформной симметрии порождают алгебру Вирасоро

$$
\left[L_{n}, L_{m}\right]=(n-m) L_{n+m}+\frac{c}{12}\left(m^{3}-m\right) \delta_{m+n, 0}
$$

где число $c$ называется центральным зарядом и является весьма важным параметром в КТП.

Пространство полей $\{A\}$ в КТП можно классифицировать в соответствии с неприводимыми представлениями алгебры Вирасоро:

$$
\{A\}=\bigoplus_{(\Delta, \bar{\Delta})}\left[\phi_{\Delta, \bar{\Delta}}\right]
$$

где $\phi_{\Delta, \bar{\Delta}}$ - основные поля:

$$
L_{n} \phi_{\Delta, \bar{\Delta}}|\varnothing\rangle=0, \quad n>0, \quad L_{0} \phi_{\Delta, \bar{\Delta}}|\varnothing\rangle=\Delta \phi_{\Delta, \bar{\Delta}}|\varnothing\rangle
$$

характеризующиеся параметром $\Delta$, который называется аномальной размерностью поля $\phi_{\Delta, \bar{\Delta}}$.

Конформная группа не исчерпывает всех симметрий в двумерных квантовых теориях поля. В настоящее время построено несколько двумерных квантовых КТП с дополнительными симметриями. Они характеризуются тем, что, кроме конформной инвариантности, содержат дополнительные бесконечномерные симметрии, порождаемые другими сохраняющимися токами. Примерами таких квантовых теорий поля являются: гиперконформная теория поля размерности $N=1$, которая в дополнение к сохраняющемуся тензору энергии-импульса $T, \bar{T}$ имеет дополнительные сохраняюшиеся токи $G, \bar{G}$ размерностей $(3 / 2,0),(0,3 / 2)$; алгебры Каца-Муди [6], порождаемые тензором энергии-импульса $T, \bar{T}$ и сохраняюшимися токами $J, \bar{J}$ размерностей $(1,0),(0,1)$; так называемые $W$-алгебры [7], являющиеся нелинейными продолжениями конформной алгебры, порождаемой полями с большими значениями спина. Основным примером $W$-алгебры является $W_{3}$-алгебра Замолодчикова [8], которая описывает квантовую конформную теорию поля с дополнительными симметриями, порождаемыми сохраняюшимися 
токами размерностей $(3,0),(0,3)$. Образуюшие $W_{3}$-алгебры удовлетворяют коммутационным соотношениям

$$
\begin{aligned}
{\left[L_{n}, W_{m}\right]=} & (n-m) W_{m+n} \\
{\left[W_{n}, W_{m}\right]=} & (n-m)\left[\frac{(n+m+2)(n+m+3)}{15}-\frac{(n+2)(m+2)}{6}\right] L_{n+m}+ \\
& +\frac{c}{3 \cdot 5 !}\left(n^{2}-4\right)\left(n^{3}-n\right) \delta_{n+m, 0}+b^{2}(n-m) \Lambda_{n+m}
\end{aligned}
$$

где

$$
b^{2}=\frac{16}{22+5 c}, \quad \Lambda_{n}=\sum_{k=-\infty}^{+\infty}: L_{k} L_{n-k}:+\frac{1}{5} \rho L_{n},
$$

а $W_{n}-$ коэффициенты Лорана сохраняюшихся токов размерности $(3,0)$ :

$$
W=\sum_{n=-\infty}^{\infty} W_{n} z^{-n-3}
$$

Следует отметить, что $W$-алгебра содержит алгебру Вирасоро как подалгебру.

Теория поля Тоды (ТПТ) дает чрезвычайно полезное описание большого класса теорий двумерных интегрируемых квантовых полей. Теории поля Тоды можно разделить на следующие три категории: конечные теории поля Тоды (КТПТ), для которых основной алгеброй является конечная алгебра Ли; афффинные теории поля Тоды, для которых основной алгеброй является аффинная алгебра; неопределенные теории поля Тоды (НТПТ), для которых основной алгеброй является неопределенная алгебра Каца-Муди. Классы КТПТ и аффинные ТПТ хорошо изучены. Известно, что они интегрируемые. Действие КТПТ задается соотношением

$$
S=\int d^{2} x\left[\frac{1}{8 \pi}(\partial \boldsymbol{\phi})^{2}+\mu \sum_{i} e^{b \mathbf{e}_{i} \cdot \boldsymbol{\phi}}\right]
$$

где $\mathbf{e}_{i}$ - простые корни алгебры Ли, $b$ - константа связи, а $\mu$ - константа, называемая космологической. Эти теории конформно-инвариантны. Кроме того, они обладают высшими симметриями, описываемыми $W$-алгебрами.

В следуюшем разделе мы получим минимальную модель $W$-алгебры на гиперэллиптических римановых поверхностях. Затем в разделе 3 мы покажем, что, используя гипотезу Полякова [9], можно свести функцию распределения ТПТ на некоторой ГЭП к произведению двух функций распределения: теории “свободного поля" на сфере с включенными вершинными операторами Тоды и теории свободного скалярного поля с антипериодическими граничными условиями и включенными полями твистов. 


\section{W-АЛГЕБРА НА ГЭП}

ГЭП $\Gamma_{g}$ является компактной римановой поверхностью рода $g \geqslant 1$ и задается алгебраическим уравнением вида

$$
y^{2}(z)=P_{2 g+2}(z)=\prod_{i=1}^{2 g+2}\left(z-x_{i}\right),
$$

где $P_{2 g+2}(z)$ - полином степени $2 g+2$. Другими словами, $\Gamma_{g}-$ это двулистное разветвленное накрытие римановой сферы. Следует заметить, что любая риманова поверхность рода $g=1$ или $g=2$ имеет вид (4), тогда как в случае рода $g \geqslant 3$ это не так.

Нашей целью является изучение гиперэллиптической формулировки $W$-алгебры и моды Тоды. Фактически это означает, что мы сведем $W$-алгебры и модели Тоды на ГЭП к эквивалентным моделям $W$-алгебры и моделям Тоды на плоскости с нетривиальными граничными условиями.

При гиперэллиптическом отображении (4) или, что эквивалентно, при отображении

$$
y^{(k)}(z)=(-)^{k} \prod_{i=1}^{2 g+2}\left(z-x_{i}\right)^{1 / 2}, \quad k=0,1
$$

любое поле $\phi_{\Delta, \bar{\Delta}}(y)$ в КТП на $\Gamma_{g}$ отображается в два поля

$$
\phi^{(k)}(z)=\left(\frac{d y^{(k)}}{d z}\right)^{\Delta} \phi\left(y^{(k)}\right), \quad k=0,1
$$

Каждая компонента поля существует на соответствующем римановом листе. Кроме того, мы должны учитывать свойства монодромии полей в соответствии с $\mathbb{Z}_{2}$-структурой ГЭП [10]; в частности, наше построение будет довольно близко следовать построениям Крнковича и других. Следует различать два типа монодромического поведения: один в точках ветвления и другой вне этих точек. При аналитических преобразованиях

$$
\pi_{x} z=(z-x) e^{2 \pi i}+x, \quad \pi_{x} y^{(k)}=y^{(k+1)}
$$

получаются следующие граничные условия для квантовых полей:

$$
\begin{aligned}
\pi_{x} \phi^{(0)}(z) & =e^{-2 \pi i(\Delta-\bar{\Delta})} \phi^{(1)}(z), & \pi_{x} \phi^{(1)}(z) & =\phi^{(0)}(z), \\
\pi \phi^{(k)}(z) & =e^{-2 \pi i(\Delta-\bar{\Delta})} \phi^{(k)}(z), & k & =0,1 .
\end{aligned}
$$

При преобразовании (5) каждый из сохраняющихся токов $T(y), W(y)$ на $\Gamma_{g}$ также отображается на два поля $T^{(k)}, W^{(k)}, k=0,1$. Соответствуюшие операторные уравнения 
можно легко выписать, поэтому здесь мы их опускаем. Эти операторные уравнения дополняются свойствами монодромии образующих $T^{(k)}, W^{(k)}, k=0,1$ :

$$
\begin{aligned}
\pi_{x} T^{(0)}(z) & =T^{(1)}(z), & \pi_{x} T^{(1)}(z) & =T^{(0)}(z), \\
\pi_{x} W^{(0)}(z) & =W^{(1)}(z), & \pi_{x} W^{(1)}(z) & =W^{(0)}(z), \\
\pi T^{(k)}(z) & =T^{(k)}(z), & \pi W^{(k)}(z) & =W^{(k)}(z) .
\end{aligned}
$$

Более полезно перейти $\mathrm{k} \mathbb{Z}_{2}$-диагональному базису. Введем определения

$$
\begin{array}{rlrl}
T & \equiv T^{(0)}+T^{(1)}, & \widetilde{T} & \equiv T^{(0)}-T^{(1)} \\
W & \equiv W^{(0)}+W^{(1)}, \quad \widetilde{W} \equiv W^{(0)}-W^{(1)} .
\end{array}
$$

В этом базисе свойства монодромии имеют вид

$$
\pi T=T, \quad \pi \widetilde{T}=\widetilde{T}, \quad \pi W=W, \quad \pi \widetilde{W}=\widetilde{W}
$$

для $\pi$ и

$$
\pi_{x} T=T, \quad \pi_{x} \widetilde{T}=-\widetilde{T}, \quad \pi_{x} W=W, \quad \pi_{x} \widetilde{W}=-\widetilde{W}
$$

для $\pi_{x}$. Заметим, что они очевидным образом идентифицируют скрученный и нескрученный секторы, соответственно.

Операторные уравнения для новых образующих легко получаются с помощью непосредственных вычислений. Мы оставляем это простое упражнение читателю. Чтобы записать эту алгебру в коммутаторной форме, выпишем разложение $T, \widetilde{T}, W, \widetilde{W}$ по модам:

$$
\begin{aligned}
T(z) V_{l}(0) & =\sum_{n \in \mathbb{Z}} z^{n-2} L_{-n} V_{l}(0), & \widetilde{T}(z) V_{l}(0) & =\sum_{m \in \mathbb{Z}} z^{m-2-l / 2} \tilde{L}_{-m+l / 2} V_{l}(0), \\
W(z) V_{l}(0) & =\sum_{n \in \mathbb{Z}} z^{n-3} W_{-n} V_{l}(0), & \widetilde{W}(z) V_{l}(0) & =\sum_{m \in \mathbb{Z}} z^{m-3-l / 2} \widetilde{W}_{-m+l / 2} V_{l}(0)
\end{aligned}
$$

где $l \in\{0,1\}$ различает два сектора: $l=0$ относится к нескрученному сектору, a $l=1-$ к скрученному. Затем, проводя стандартные вычисления, получим следующие коммутационные соотношения для операторов $L_{n}, \tilde{L}_{n+l / 2}, W_{n}, \widetilde{W}_{n+l / 2}$ :

$$
\begin{aligned}
{\left[L_{n}, L_{m}\right]=} & (n-m) L_{n+m}+\frac{c}{12}\left(m^{3}-m\right) \delta_{m+n, 0} \\
{\left[L_{n}, W_{m}\right]=} & (n-m) W_{m+n}, \\
{\left[W_{n}, W_{m}\right]=} & (n-m)\left[\frac{(n+m+2)(n+m+3)}{15}-\frac{(n+2)(m+2)}{6}\right] L_{n+m}+ \\
& +\frac{c}{3 \cdot 5 !}\left(n^{2}-4\right)\left(n^{3}-n\right) \delta_{n+m, 0}+b^{2}(n-m) \Lambda_{n+m}
\end{aligned}
$$




$$
\begin{aligned}
{\left[\tilde{L}_{n+l / 2} \tilde{L}_{m+l / 2}\right]=} & (n-m) L_{n+m+l}+\frac{\hat{c}}{12}\left[\left(n+\frac{l}{2}\right)^{3}-\left(n+\frac{l}{2}\right)\right] \delta_{n+m+l, 0}, \\
{\left[L_{n}, \tilde{L}_{m+l / 2}\right]=} & \left(n-m-\frac{l}{2}\right) \tilde{L}_{m+n+l / 2}, \\
{\left[\tilde{L}_{n+l / 2}, W_{m}\right]=} & (2 n+l-m) \widetilde{W}_{m+n+l / 2}, \\
{\left[L_{n}, \widetilde{W}_{m+l / 2}\right]=} & \left(2 n-m-\frac{l}{2}\right) \widetilde{W}_{m+n+l / 2}, \\
{\left[\tilde{L}_{n+l / 2}, \widetilde{W}_{m+l / 2}\right]=} & \left(2 n-m+\frac{l}{2}\right) W_{m+n}, \\
\left.\widetilde{W}_{n+l / 2}, \widetilde{W}_{m+l / 2}\right]= & (n-m)\left[\frac{1}{15}(n+m+l+2)(n+m+l+3)-\right. \\
& \left.-\frac{1}{6}\left(n+\frac{l}{2}+2\right)\left(m+\frac{l}{2}+2\right)\right] L_{n+m+l}+b^{2}(n-m) \Lambda_{n+m+l}+ \\
& +\frac{\hat{c}}{3 \cdot 5 !}\left[\left(n+\frac{l}{2}\right)^{2}-4\right]\left[\left(n+\frac{l}{2}\right)^{3}-\left(n+\frac{l}{2}\right)\right] \delta_{n+m+l, 0}, \\
{\left[W_{n}, \widetilde{W}_{m+l / 2}\right]=} & \left(n-m-\frac{l}{2}\right)\left[\frac{1}{15}\left(n+m+\frac{l}{2}+2\right)\left(n+m+\frac{l}{2}+3\right)-\right. \\
& \left.-\frac{1}{6}(n+2)\left(m+\frac{l}{2}+2\right)\right] \tilde{L}_{n+m+l / 2}+b^{2}\left(n-m-\frac{l}{2}\right) \tilde{\Lambda}_{n+m+l / 2} .
\end{aligned}
$$

Эти коммутационные соотношения образуют $W$-алгебру на ГЭП $W_{\Gamma}$.

Пространство полей $\{A\}$ можно разложить на два подпространства: одно - скрученное $\left\{A_{1}\right\}$, другое - нескрученное $\left\{A_{0}\right\}$, которые можно далее классифицировать в соответствии с неприводимыми представлениями $W$-алгебры на ГЭП:

$$
\{A\}=\left\{A_{0}\right\}+\left\{A_{1}\right\}=\bigoplus_{i}\left[V_{0 i}\right]+\bigoplus_{i}\left[V_{1 i}\right] .
$$

Подпространства $\left[V_{l, i}\right]$ образуют построенный над основным полем $V_{l, i}$ модуль, удовлетворяюший уравнениям

$$
\begin{aligned}
& L_{0} V_{0 i}=\Delta_{i} V_{0 i}, \quad \tilde{L}_{0} V_{0 i}=\tilde{\Delta}_{i} V_{0 i}, \quad L_{n} V_{0 i}=\tilde{L}_{n} V_{0 i}=0, \quad n>0, \\
& W_{0} V_{0 i}=\omega_{i} V_{0 i}, \quad \widetilde{W}_{0} V_{0 i}=\widetilde{\omega}_{i} V_{0 i}, \quad W_{n} V_{0 i}=\widetilde{W}_{n} V_{0 i}=0, \quad n>0,
\end{aligned}
$$

в нескрученном секторе и

$$
\begin{aligned}
& L_{0} V_{1 i}=\Delta_{i} V_{1 i}, \quad L_{n} V_{1 i}=\tilde{L}_{n-1 / 2} V_{1 i}=0, \quad n>0, \\
& W_{0} V_{1 i}=\omega_{i} V_{1 i}, \quad W_{n} V_{1 i}=\widetilde{W}_{n-1 / 2} V_{1 i}=0, \quad n>0,
\end{aligned}
$$

в скрученном секторе. 
Доценко и Фатеев [11] развили мощный метод, известный как представление кулоновского газа квантовой конформной модели на сфере, который дает полное решение для коррелящионных функций в минимальной модели на сфере. Они смогли написать интегральные представления конформных блоков через корреляционные функции вершинных операторов свободного бозонного скалярного поля $\varphi$ с фоновым зарядом $\alpha_{0}$. Мы обобщим этот метод на случай $W$-алгебры на ГЭП. Для этого введем двухкомпонентное свободное безмассовое бозонное поле $\varphi^{(k)}=\left(\varphi_{1}^{(k)}, \varphi_{2}^{(k)}\right)$ на каждом листе $k=0,1$. Можно проверить, что представление кулоновского газа

$$
\begin{aligned}
T^{(k)}= & -\frac{1}{4}:\left(\partial \varphi_{1}^{(k)}\right)^{2}:-\frac{1}{4}:\left(\partial \varphi_{2}^{(k)}\right)^{2}:+i \alpha_{0} \partial^{2} \varphi_{1}^{(k)}, \\
\frac{12 i}{b} W^{(k)}= & :\left(\partial \varphi_{2}^{(k)}\right)^{3}:-3:\left(\partial \varphi_{1}^{(k)}\right)^{2} \partial \varphi_{2}^{(k)}:+3 i \alpha_{0}: \partial^{2} \varphi_{1}^{(k)} \partial \varphi_{2}^{(k)}:+ \\
& +9 i \alpha_{0}: \partial \varphi_{1}^{(k)} \partial^{2} \varphi_{2}^{(k)}:+6 \alpha_{0}^{2} \partial^{3} \varphi_{2}^{(k)}
\end{aligned}
$$

при $c=2-24 \alpha_{0}^{2}$ удовлетворяет всем требуемым операторным разложениям, если задан пропагатор

$$
\left\langle\varphi_{i}^{\left(k_{1}\right)}(z) \varphi_{j}^{\left(k_{2}\right)}\left(z^{\prime}\right)\right\rangle=-2 \delta_{i j} \delta_{k_{1} k_{2}} \ln \left(z-z^{\prime}\right), \quad k_{1}, k_{2} \in\{0,1\} .
$$

Поля также удовлетворяют условиям

$$
\pi_{x} \partial \varphi_{i}^{(0)}=\partial \varphi_{i}^{(1)}, \quad \pi_{x} \partial \varphi_{i}^{(1)}=\partial \varphi_{i}^{(0)}, \quad \pi \partial \varphi_{i}^{(k)}=\partial \varphi_{i}^{(k)} .
$$

Запишем поля в базисе, в котором операторы являются диагональными:

$$
\begin{gathered}
\varphi_{i}=\varphi_{i}^{(0)}+\varphi_{i}^{(1)}, \quad \tilde{\varphi}_{i}=\varphi_{i}^{(0)}-\varphi_{i}^{(1)} \\
\pi_{x} \partial \varphi_{i}=\partial \varphi_{i}, \quad \pi_{x} \partial \tilde{\varphi}_{i}=-\partial \tilde{\varphi}_{i}, \quad \pi \partial \varphi_{i}=\partial \varphi_{i}, \quad \pi \partial \tilde{\varphi}_{i}=\partial \tilde{\varphi}_{i}, \\
\left\langle\varphi_{i}(z) \varphi_{j}\left(z^{\prime}\right)\right\rangle=\left\langle\tilde{\varphi}_{i}(z) \tilde{\varphi}_{j}\left(z^{\prime}\right)\right\rangle=-4 \delta_{i j} \ln \left(z-z^{\prime}\right) .
\end{gathered}
$$

Сравнивая свойства этих полей со свойствами объемных свободных полей на сфере, сразу обнаружим нетривиальные монодромические свойства, определяемые структурой ГЭП. В терминах новых свободных бозонных полей образующие токи $W$-алгебры можно записать в виде

$$
\begin{aligned}
T= & -\frac{1}{8}:\left(\partial \varphi_{1}\right)^{2}:-\frac{1}{8}:\left(\partial \varphi_{2}\right)^{2}:-\frac{1}{8}:\left(\partial \tilde{\varphi}_{1}\right)^{2}:-\frac{1}{8}:\left(\partial \tilde{\varphi}_{2}\right)^{2}:+i \alpha_{0} \partial^{2} \varphi_{1}, \\
\widetilde{T}= & -\frac{1}{4}: \partial \tilde{\varphi}_{1} \partial \varphi_{1}:-\frac{1}{4}: \partial \tilde{\varphi}_{2} \partial \varphi_{2}:+i \alpha_{0} \partial^{2} \tilde{\varphi}_{1}, \\
\frac{24 i}{b} W= & \frac{1}{2}:\left(\partial \varphi_{2}\right)^{3}:-\frac{3}{2}\left[:\left(\partial \varphi_{1}\right)^{2} \partial \varphi_{2}:+:\left(\partial \tilde{\varphi}_{1}\right)^{2} \partial \varphi_{2}:-:\left(\partial \tilde{\varphi}_{2}\right)^{2} \partial \varphi_{2}:\right]+ \\
& +3 i \alpha_{0}\left[: \partial^{2} \varphi_{1} \partial \varphi_{2}:+: \partial^{2} \tilde{\varphi}_{1} \partial \tilde{\varphi}_{2}:\right]+9 i \alpha_{0}\left[: \partial \varphi_{1} \partial^{2} \varphi_{2}:+: \partial \tilde{\varphi}_{1} \partial^{2} \tilde{\varphi}_{2}:\right]- \\
& -3: \partial \varphi_{1} \partial \tilde{\varphi}_{1} \partial \tilde{\varphi}_{2}:+12 \alpha_{0}^{2} \partial^{3} \varphi_{2}, \\
\frac{24 i}{b} \widetilde{W}= & \frac{1}{2}:\left(\partial \tilde{\varphi}_{2}\right)^{3}:-\frac{3}{2}\left[:\left(\partial \varphi_{1}\right)^{2} \partial \tilde{\varphi}_{2}:+:\left(\partial \tilde{\varphi}_{1}\right)^{2} \partial \tilde{\varphi}_{2}:-:\left(\partial \varphi_{2}\right)^{2} \partial \tilde{\varphi}_{2}:\right]+ \\
& +3 i \alpha_{0}\left[: \partial^{2} \varphi_{1} \partial \tilde{\varphi}_{2}:+: \partial^{2} \tilde{\varphi}_{1} \partial \varphi_{2}:\right]+9 i \alpha_{0}\left[: \partial \varphi_{1} \partial^{2} \tilde{\varphi}_{2}:+: \partial \tilde{\varphi}_{1} \partial^{2} \varphi_{2}:\right]- \\
& -3: \partial \varphi_{1} \partial \tilde{\varphi}_{1} \partial \varphi_{2}:+12 \alpha_{0}^{2} \partial^{3} \tilde{\varphi}_{2},
\end{aligned}
$$


где центральный заряд имеет вид $\hat{c}=4-48 \alpha_{0}^{2}$. Анализируя величину центрального заряда и представление токов через свободные бозоны, можно заметить, что фактически мы имеем составную теорию, которая распадается в прямую сумму двух теорий: одна - теория бозонного свободного поля на сфере с периодическими граничными условиями и центральным зарядом $\hat{c}_{s}=2-48 \alpha_{0}^{2}$, другая - теория бозонного свободного поля с антипериодическими граничными условиями и центральным зарядом $\hat{c}_{o}=2$.

Теперь мы построим представления примарных полей для каждого подпространства, используя вершинные операторы бозонных полей.

В нескрученном подпространстве вершинные операторы вида $V_{0}=e^{i \mathbf{a} \cdot \boldsymbol{\varphi}+i \mathbf{b} \cdot \boldsymbol{\varphi}}$ имеют аномальные размерности

$$
\Delta=2 \mathbf{b}^{2}+2 \mathbf{a} \cdot\left(\mathbf{a}-2 \boldsymbol{\alpha}_{0}\right), \quad \tilde{\Delta}=4 \mathbf{b} \cdot\left(\mathbf{a}-\boldsymbol{\alpha}_{0}\right)
$$

и веса

$$
\begin{aligned}
& \omega=\frac{4}{3} b\left[a_{2}^{3}-3\left(a_{1}^{2} a_{2}+b_{1}^{2} a_{2}-b_{2}^{2} a_{2}\right)+6 \alpha_{0}\left(a_{1} a_{2}+b_{1} b_{2}\right)-6 a_{1} b_{1} b_{2}-3 \alpha_{0}^{2} a_{2}\right], \\
& \widetilde{\omega}=\frac{4}{3} b\left[b_{2}^{3}-3\left(a_{1}^{2} b_{2}+b_{1}^{2} b_{2}-a_{2}^{2} b_{2}\right)+6 \alpha_{0}\left(a_{1} b_{2}+b_{1} a_{2}\right)-6 a_{1} b_{1} a_{2}-3 \alpha_{0}^{2} b_{2}\right] .
\end{aligned}
$$

В этих выражениях мы полагаем, что $\boldsymbol{\alpha}_{0}=\left(\alpha_{0}, 0\right)$.

Подпространство $\left[V_{0 i}\right]$ порождается всеми возможными векторами вида

$$
L_{-n_{1}} \ldots L_{-n_{k}} \tilde{L}_{-n_{1}^{\prime}} \ldots \tilde{L}_{-n_{k}^{\prime}} W_{-m_{1}} \ldots W_{-m_{k}} \widetilde{W}_{-m_{1}^{\prime}} \ldots \widetilde{W}_{-m_{k}^{\prime}} V_{0 i}|\varnothing\rangle,
$$

где $n, n^{\prime}, m, m^{\prime}>0$.

Заметим, что для размерностей и весов основных полей выполняются следующие соотношения симметрии:

$$
\begin{array}{ll}
\Delta\left(2 \boldsymbol{\alpha}_{0}-\mathbf{a}, \mathbf{b}\right)=\Delta\left(\mathbf{a}^{*}, \mathbf{b}^{*}\right)=\Delta(\mathbf{a}, \mathbf{b}), & \tilde{\Delta}\left(2 \boldsymbol{\alpha}_{0}-\mathbf{a}, \mathbf{b}\right)=\tilde{\Delta}\left(\mathbf{a}^{*}, \mathbf{b}^{*}\right)=-\tilde{\Delta}(\mathbf{a}, \mathbf{b}), \\
\omega\left(2 \boldsymbol{\alpha}_{0}-\mathbf{a}, \mathbf{b}\right)=\omega\left(\mathbf{a}^{*}, \mathbf{b}^{*}\right)=-\omega(\mathbf{a}, \mathbf{b}), & \widetilde{\omega}\left(2 \boldsymbol{\alpha}_{0}-\mathbf{a}, \mathbf{b}\right)=\widetilde{\omega}\left(\mathbf{a}^{*}, \mathbf{b}^{*}\right)=\widetilde{\omega}(\mathbf{a}, \mathbf{b}),
\end{array}
$$

где $\mathbf{a}^{*}=\left(a_{1},-a_{2}\right)$ и $\mathbf{b}^{*}=\left(-b_{1}, b_{2}\right)$. Из этих соотношений симметрии следует, что пространство $\left[V_{\mathbf{a}, \mathbf{b}}\right]$ эквивалентно пространству $\left[V_{2 \boldsymbol{\alpha}_{0}-\mathbf{a}^{*}, \mathbf{b}^{*}}\right]$.

В скрученном подпространстве ситуация несколько иная. Здесь мы имеем антипериодическое бозонное поле $\partial \tilde{\varphi}$. Антипериодические граничные условия приводят к деформации геометрии, антипериодические свойства тока $\partial \tilde{\varphi}$ в операторных уравнениях обеспечиваются введением рамоновского поля твиста $\sigma(z)$ [12]. Более подробно, операторное произведение тока $\tilde{I}=i \partial \tilde{\varphi}$ на поле $\sigma$ имеет вид

$$
\tilde{I}(z) \sigma(0)=\frac{1}{2} z^{-1 / 2} \hat{\sigma}(0)+\cdots,
$$


где $\hat{\sigma}=\tilde{I}_{-1 / 2} \sigma$ и

$$
\tilde{I}(z) \hat{\sigma}(0)=\frac{1}{2} z^{-3 / 2} \sigma(0)+2 z^{-1 / 2} \sigma^{\prime}(0)+\cdots .
$$

Поля $\sigma$ и $\hat{\sigma}$ - это примарные поля по отношению к тензору энергии-импульса

$$
T_{\mathrm{orb}}=-\frac{1}{8}\left(\partial \tilde{\varphi}_{1}\right)^{2}-\frac{1}{8}\left(\partial \tilde{\varphi}_{2}\right)^{2}
$$

с аномальными размерностями $\Delta=1 / 16$ и $\hat{\Delta}=9 / 16$, соответственно.

В скрученном секторе можно записать примарные поля в виде

$$
V_{1}=e^{i \mathbf{d} \cdot \boldsymbol{\varphi}} \sigma
$$

с аномальными размерностями и весом

$$
\begin{aligned}
& \Delta=2 \mathbf{d}\left(\mathbf{d}-2 \boldsymbol{\alpha}_{0}\right)+\frac{1}{8} \\
& \omega=\frac{4}{3} d_{2} b\left(d_{2}^{2}-3 d_{1}^{2}+6 \alpha_{0} d_{1}-3 \alpha_{0}^{2}\right)+\frac{b}{4}\left(\frac{d_{2}}{8}+\frac{d_{1}}{2}-b \alpha_{0}\right) .
\end{aligned}
$$

Легко заметить симметрию

$$
\Delta\left(2 \boldsymbol{\alpha}_{0}-\mathbf{d}\right)=\Delta\left(\mathbf{d}^{*}\right)=\Delta(\mathbf{d})
$$

Операторные разложения примарных полей с $T(z)$ и $W(z)$ имеют вид

$$
\begin{aligned}
\widetilde{T}(z) V(0) & =\frac{i}{4}\left(2 d_{1}+2 d_{2}-\alpha_{0}\right) \frac{1}{z^{3 / 2}} \widehat{V}(0)+\cdots \\
\widetilde{W}(z) V(0) & =\frac{b}{8 i}\left(4 d_{1}^{2}-4 d_{2}^{2}-\frac{3}{8} \frac{1}{16}-5 \alpha_{0} d_{1}-7 \alpha_{0} d_{2}+8 d_{1} d_{2}+\frac{3}{2} \alpha_{0}^{2}\right) \frac{1}{z^{5 / 2}} \widehat{V}(0)+\cdots
\end{aligned}
$$

(здесь выписаны только главные члены).

Подмодуль $\left[V_{1 i}\right]$ порождается всеми возможными векторами вида

$L_{-n_{1}} \ldots L_{-n_{k}} \tilde{L}_{-n_{1}^{\prime}+1 / 2} \ldots \tilde{L}_{-n_{k}^{\prime}+1 / 2} W_{-m_{1}} \ldots W_{-m_{k}} \widetilde{W}_{-m_{1}^{\prime}+1 / 2} \ldots \widetilde{W}_{-m_{k}^{\prime}+1 / 2} V_{1 i}|\varnothing\rangle$ где $n, n^{\prime}, m, m^{\prime}>0$.

Итак, мы построили неприводимое представление алгебры $\{A\}$ в пространстве Фока:

$$
\begin{aligned}
:\left\{\left(\partial \varphi_{1}\right)^{s_{1}}\left(\partial^{2} \varphi_{1}\right)^{s_{2}} \ldots\right\}\left\{\left(\partial \varphi_{2}\right)^{s_{1}^{\prime}}\left(\partial^{2} \varphi_{2}\right)^{s_{2}^{\prime}} \ldots\right\} \times \\
\quad \times\left\{\left(\partial \tilde{\varphi}_{1}\right)^{l_{1}}\left(\partial^{2} \tilde{\varphi}_{1}\right)^{l_{2}} \ldots\right\}\left\{\left(\partial \tilde{\varphi}_{2}\right)^{l_{1}^{\prime}}\left(\partial^{2} \tilde{\varphi}_{2}\right)^{l_{2}^{\prime}} \ldots\right\} V_{0 i}:+ \\
+:\left\{\left(\partial \varphi_{1}\right)^{s_{1}}\left(\partial^{2} \varphi_{1}\right)^{s_{2}} \ldots\right\}\left\{\left(\partial \varphi_{2}\right)^{s_{1}^{\prime}}\left(\partial^{2} \varphi_{2}\right)^{s_{2}^{\prime}} \ldots\right\} \times \\
\quad \times\left\{\left(\partial \tilde{\varphi}_{1}\right)^{l_{1}}\left(\partial^{2} \tilde{\varphi}_{1}\right)^{l_{2}} \ldots\right\}\left\{\left(\partial \tilde{\varphi}_{2}\right)^{l_{1}^{\prime}}\left(\partial^{2} \tilde{\varphi}_{2}\right)^{l_{2}^{\prime}} \ldots\right\} V_{1 i}:
\end{aligned}
$$


В своей замечательной работе Белавин, Поляков и Замолодчиков [13] показали, как можно получить полное описание операторов (или, что эквивалентно, аномальных размерностей) в любой двумерной КТП. В частности, было дано доказательство сушествования специального дискретного класса двумерных КТП (так называемых вырожденных КТП). Далее, было показано, что спектр аномальных размерностей операторов, соответствующих этим теориям, задается формулой Каца. Последующее развитие этой идеи доказало, что вырожденные КТП, содержашие конечное число примарных полей, описывают критические флуктуации двумерных статистических моделей в точке фазового перехода второго рода. Эти КТП стали называть минимальными моделями. Подобным же образом мы найдем полное описание спектра аномальных размерностей для искомой $W_{\Gamma}$-алгебры. Точнее, представление $[V]$, соответствуюшее $W_{\Gamma}$, является вырожденным, если модуль $[V]$ содержит нулевой вектор $\chi_{N}$, удовлетворяюший уравнениям

$$
L_{n} \chi_{N}=W_{n} \chi_{N}=0, \quad n>0, \quad L_{0} \chi_{N}=(\Delta+N) \chi_{N} .
$$

Чтобы получить спектр зарядов $\mathbf{a}, \mathbf{b}$, соответствуюших вырожденным модулям, мы построим экранируюшие заряды, соответствующие данной теории:

$$
Q_{i}^{ \pm}=\oint_{c} d z V_{i}^{ \pm}(z)=\oint_{c} d z e^{i a_{ \pm} \mathbf{e}_{i} \cdot(\boldsymbol{\varphi}+\tilde{\boldsymbol{\varphi}})}
$$

где

$$
\mathbf{e}_{1}=(1, \sqrt{3}), \quad \mathbf{e}_{2}=(1,-\sqrt{3}), \quad a_{ \pm}=\frac{\alpha_{0}}{8} \pm \frac{1}{8} \sqrt{\alpha_{0}^{2}+4},
$$

и будем изучать условия, при которых вектор

$$
\chi_{n}=\oint_{c_{1}} d z_{1} V_{i}^{+}\left(z_{1}\right) \oint_{c_{2}} d z_{2} V_{i}^{+}\left(z_{2}\right) \cdots \oint_{c_{n}} d z_{n} V_{i}^{+}\left(z_{n}\right) V_{2 \boldsymbol{\alpha}_{0}-\mathbf{a}^{*}-n \mathbf{e}_{i} a_{+}, \mathbf{b}^{*}-n \mathbf{e}_{i} a_{+}}
$$

становится нулевым. Если принять во внимание операторное разложение

$$
\begin{aligned}
& V_{i}^{+}(z) V_{2 \boldsymbol{\alpha}_{0}-\mathbf{a}^{*}-n \mathbf{e}_{i} a_{+}, \mathbf{b}^{*}-n \mathbf{e}_{i} a_{+}}(0)= \\
& \quad=z^{4 a_{+} \mathbf{e}_{i}\left(2 \boldsymbol{\alpha}_{0}-\mathbf{a}^{*}-n \mathbf{e}_{i} a_{+}\right)+4 a_{+} \mathbf{e}_{i}\left(\mathbf{b}^{*}-n \mathbf{e}_{i} a_{+}\right)}: V_{i}^{+} V_{2 \boldsymbol{\alpha}_{0}-\mathbf{a}^{*}-n \mathbf{e}_{i} a_{+}, \mathbf{b}^{*}-n \mathbf{e}_{i} a_{+}}:
\end{aligned}
$$

то можно показать, что интеграл (9) не равен нулю, если уравнение

$$
16 a_{+}^{2}(n-1)+4 a_{+} \mathbf{e}_{i} \cdot\left(2 \boldsymbol{\alpha}_{0}-\mathbf{a}^{*}-n \mathbf{e}_{i} a_{+}\right)+4 a_{+} \mathbf{e}_{i} \cdot\left(\mathbf{b}^{*}-n \mathbf{e}_{i} a_{+}\right)=-m-1
$$

выполняется при некотором положительном целом $m$. Решение этого алгебраического уравнения приводит к следуюшим выражениям для зарядов $\mathbf{a}, \mathbf{b}$ :

$$
\begin{aligned}
& \mathbf{a}\left(\begin{array}{l}
n, m \\
n^{\prime}, m^{\prime}
\end{array}\right)=2 \boldsymbol{\omega}_{1}\left[(3-n) a_{+}+(3-m) a_{-}\right]+2 \boldsymbol{\omega}_{2}\left[\left(3-n^{\prime}\right) a_{+}+\left(3-m^{\prime}\right) a_{-}\right], \\
& \mathbf{b}\left(\begin{array}{l}
n, m \\
n^{\prime}, m^{\prime}
\end{array}\right)=2 \boldsymbol{\omega}_{1}\left[(1+n) a_{+}+(1+m) a_{-}\right]+2 \boldsymbol{\omega}_{2}\left[\left(1+n^{\prime}\right) a_{+}+\left(1+m^{\prime}\right) a_{-}\right],
\end{aligned}
$$


где

$$
\boldsymbol{\omega}_{1}=\left(\frac{1}{2}, \frac{\sqrt{3}}{2}\right), \quad \omega_{2}=\left(\frac{1}{2},-\frac{\sqrt{3}}{2}\right)
$$

Эти формулы полностью определяют вырожденные нескрученные подмодули $W_{\Gamma^{-}}$ алгебры. Соответствующий спектр Каца параметров $\Delta\left(\begin{array}{l}n m \\ n^{\prime} m^{\prime}\end{array}\right), \tilde{\Delta}\left(\begin{array}{c}n m \\ n^{\prime} m^{\prime}\end{array}\right), \omega\left(\begin{array}{c}n m \\ n^{\prime} m^{\prime}\end{array}\right)$, $\widetilde{\omega}\left(\begin{array}{l}n m \\ n^{\prime} m^{\prime}\end{array}\right)$ можно легко построить по формулам (6) и (7).

Аналогично можно найти спектр зарядов для скрученного подмодуля:

$$
\begin{aligned}
& d_{1}\left(\begin{array}{l}
n, m \\
n^{\prime}, m^{\prime}
\end{array}\right)=2\left[\left(4-n-n^{\prime}\right) a_{+}+\left(4-m-m^{\prime}\right) a_{-}\right] \\
& d_{2}\left(\begin{array}{l}
n, m \\
n^{\prime}, m^{\prime}
\end{array}\right)=\frac{1}{\sqrt{3}}\left[\left(n^{\prime}-n\right) a_{+}+\left(m^{\prime}-m\right) a_{-}\right] .
\end{aligned}
$$

Соответствуюшие параметры $\Delta\left(\begin{array}{l}n m \\ n^{\prime} m^{\prime}\end{array}\right), \omega\left(\begin{array}{l}n m \\ n^{\prime} m^{\prime}\end{array}\right)$, определяющие спектр Каца вырожденного скрученного подмодуля, вычисляются по формулам (8).

Теперь можно выписать формулы для корреляционных функций и функций распределения на ГЭП. $N$-точечные корреляционные функции

$$
G_{\Gamma}\left(x_{i}, z_{j}\right)=\left\langle\prod_{i=1}^{2 g+2} V_{1}\left[\begin{array}{l}
1,1 \\
1,1
\end{array}\right]\left(x_{i}\right) \prod_{j=1}^{N} V_{0}\left[\begin{array}{c}
n_{j}, m_{j} \\
n_{j}^{\prime}, m_{j}^{\prime}
\end{array}\right]\left(z_{j}\right)\left(Q_{1}^{+}\right)^{s}\left(Q_{1}^{-}\right)^{t}\left(Q_{2}^{+}\right)^{r}\left(Q_{2}^{-}\right)^{p}\right\rangle
$$

задаются контурными интегралами

$$
\begin{aligned}
G_{\Gamma}\left(x_{i}, z_{j}\right)= & \left\langle\prod_{i=1}^{2 g+2} V_{1}\left[\begin{array}{l}
1,1 \\
1,1
\end{array}\right]\left(x_{i}\right) \prod_{j=1}^{N} V_{0}\left[\begin{array}{c}
n_{j}, m_{j} \\
n_{j}^{\prime}, m_{j}^{\prime}
\end{array}\right]\left(z_{j}\right) \times\right. \\
& \times \oint d z_{1} V_{1}^{+}\left(z_{1}\right) \cdots \oint d z_{n} V_{1}^{+}\left(z_{s}\right) \oint d z_{1}^{\prime} V_{1}^{-}\left(z_{1}^{\prime}\right) \cdots \oint d z_{t}^{\prime} V_{1}^{-}\left(z_{t}^{\prime}\right) \times \\
& \left.\times \oint d \zeta_{1} V_{2}^{+}\left(\zeta_{1}\right) \cdots \oint d \zeta_{r} V_{2}^{+}\left(\zeta_{r}\right) \oint d \zeta_{1}^{\prime} V_{1}^{-}\left(\zeta_{1}^{\prime}\right) \cdots \oint d \zeta_{p}^{\prime} V_{1}^{-}\left(\zeta_{p}^{\prime}\right)\right\rangle,
\end{aligned}
$$

где подынтегральное выражение, конечно, является многозначной функцией и определено на римановых поверхностях. Корреляционная функция не обращается в нуль, только если заряды удовлетворяют уравнениям

$$
\begin{aligned}
& \sum_{i=1}^{g+1} \mathbf{d}_{i}+\sum_{j=1}^{N} \mathbf{a}_{j}+s a_{+} \mathbf{e}_{1}+t a_{-} \mathbf{e}_{1}+r a_{+} \mathbf{e}_{2}+p a_{-} \mathbf{e}_{2}=2 \boldsymbol{\alpha}_{0} \\
& \sum_{i=1}^{g+1} \mathbf{q}_{i}+\sum_{j=1}^{N} \mathbf{b}_{j}+s a_{+} \mathbf{e}_{1}+t a_{-} \mathbf{e}_{1}+r a_{+} \mathbf{e}_{2}+p a_{-} \mathbf{e}_{2}=0 .
\end{aligned}
$$


Аналогично мы можем выписать интегральное представление для функции распределения на ГЭП:

$$
\begin{aligned}
Z_{\Gamma}\left(x_{1}, x_{2}, \ldots, x_{2 g+2}\right)=\left\langle\prod_{i=1}^{2 g+2} V_{1}\left[\begin{array}{l}
1,1 \\
1,1
\end{array}\right]\left(x_{i}\right)\left(Q_{1}^{+}\right)^{s}\left(Q_{1}^{-}\right)^{t}\left(Q_{2}^{+}\right)^{r}\left(Q_{2}^{-}\right)^{p}\right\rangle= \\
=\left\langle\prod_{i=1}^{2 g+2} V_{1}\left[\begin{array}{l}
1,1 \\
1,1
\end{array}\right]\left(x_{i}\right) \oint d z_{1} V_{1}^{+}\left(z_{1}\right) \cdots \oint d z_{n} V_{1}^{+}\left(z_{s}\right) \oint d z_{1}^{\prime} V_{1}^{-}\left(z_{1}^{\prime}\right) \cdots \oint d z_{t}^{\prime} V_{1}^{-}\left(z_{t}^{\prime}\right) \times\right. \\
\left.\quad \times \oint d \zeta_{1} V_{2}^{+}\left(\zeta_{1}\right) \cdots \oint d \zeta_{r} V_{2}^{+}\left(\zeta_{r}\right) \oint d \zeta_{1}^{\prime} V_{1}^{-}\left(\zeta_{1}^{\prime}\right) \cdots \oint d \zeta_{p}^{\prime} V_{1}^{-}\left(\zeta_{p}^{\prime}\right)\right\rangle .
\end{aligned}
$$

Эта“функция распределения” не обращается в нуль при условии, что

$$
\begin{aligned}
& \sum_{i=1}^{g+1} \mathbf{d}_{i}+s a_{+} \mathbf{e}_{1}+t a_{-} \mathbf{e}_{1}+r a_{+} \mathbf{e}_{2}+p a_{-} \mathbf{e}_{2}=2 \boldsymbol{\alpha}_{0} \\
& \sum_{i=1}^{g+1} \mathbf{q}_{i}+s a_{+} \mathbf{e}_{1}+t a_{-} \mathbf{e}_{1}+r a_{+} \mathbf{e}_{2}+p a_{-} \mathbf{e}_{2}=0
\end{aligned}
$$

Главным пунктом в вышеприведенной конструкции является то, что вся информация и свойства основной геометрии как бы закодированы в примарных разветвленных полях $V_{1}\left[\begin{array}{l}1,1 \\ 1,1\end{array}\right]\left(x_{i}\right)$ скрученного подмодуля $W_{\Gamma}$-алгебры.

\section{3. $A_{2}-$ TПT}

Рассмотрим КТПТ, соответствующую алгебре Ли $A_{2}$ с однократными связями. Локальные свойства $A_{2}$-КТПТ выводятся из плотности лагранжиана

$$
\mathcal{L}=\frac{1}{8 \pi}(\partial \boldsymbol{\phi})^{2}+\mu \sum_{i=1}^{2} e^{b \mathbf{e}_{i} \cdot \boldsymbol{\phi}}+\frac{R}{4 \pi} \boldsymbol{\alpha}_{0} \cdot \boldsymbol{\phi},
$$

где $\mathbf{e}_{i}, i=1,2,-$ простые корни алгебры Ли $A_{2}$. Соответствующие фундаментальные веса $\mathbf{w}_{i}$ алгебры Ли $A_{2}$ определяются уравнением $\mathbf{e}_{i} \cdot \mathbf{w}_{j}=\delta_{i j}$. Фоновый заряд $\boldsymbol{\alpha}_{0}$ пропорционален вектору Вейля $\boldsymbol{\rho}$ :

$$
\boldsymbol{\alpha}_{0}=\left(b+\frac{1}{b}\right) \boldsymbol{\rho}, \quad \boldsymbol{\rho}=\sum_{i=1}^{2} \mathbf{w}_{i}
$$

Локальная конформная инвариантность КТПТ с центральным зарядом $c=2+24 \alpha_{0}^{2}$ обеспечивается сушествованием голоморфных и антиголоморфных тензоров энергииимпульса $T, \bar{T}$. Хорошо известно, что, кроме стандартной конформной симметрии, КТПТ имеет дополнительную $W$-симметрию.

Теперь мы можем ответить на вопрос, как строится функция распределения модели Тоды на $\Gamma_{g}$ с помошью вершинных операторов, соответствуюших $W_{\Gamma}$. 
Согласно основной гипотезе Полякова [9] "суммирование" по гладким метрикам с включением вершинных операторов должно быть эквивалентно "суммированию" по метрикам с особенностями в точках вставок,но без вершинных операторов. Следуя этой идее, функции распределения модели Тоды на $\Gamma_{g}$

$$
Z_{\Gamma}\left(x_{1}, x_{2}, \ldots, x_{2 g+2}\right)=\int D \phi e^{-S_{\mathrm{T}}[\phi]}
$$

можно представить в виде

$$
\begin{aligned}
Z_{\Gamma}= & \int \mathcal{D} \boldsymbol{\varphi} \mathcal{D} \tilde{\boldsymbol{\varphi}} e^{-\int(\partial \boldsymbol{\varphi})^{2} /(8 \pi)+\mu \sum_{i=1}^{2} e^{b \mathbf{e}_{i} \cdot \boldsymbol{\varphi}}+R \boldsymbol{\alpha}_{0} \cdot \boldsymbol{\varphi} /(4 \pi)+(\partial \tilde{\boldsymbol{\varphi}})^{2} /(8 \pi)} \prod_{i=1}^{2 g+2} e^{\mathbf{d} \cdot \boldsymbol{\varphi}} \sigma\left(x_{i}\right)= \\
= & \int \mathcal{D} \boldsymbol{\varphi} e^{-\int(\partial \boldsymbol{\varphi})^{2} /(8 \pi)+\mu \sum_{i=1}^{2} e^{b \mathbf{e}_{i} \cdot \boldsymbol{\varphi}}+R \boldsymbol{\alpha}_{0} \cdot \boldsymbol{\varphi} /(4 \pi)} \prod_{i=1}^{2 g+2} e^{\mathbf{d} \cdot \boldsymbol{\varphi}\left(x_{i}\right)} \times \\
& \times \int \mathcal{D} \tilde{\boldsymbol{\varphi}} e^{-\int(\partial \tilde{\boldsymbol{\varphi}})^{2} /(8 \pi)} \prod_{i=1}^{2 g+2} \sigma\left(x_{i}\right) .
\end{aligned}
$$

Введем ортогональное разложение свободного поля $\varphi$

$$
\varphi=\varphi_{\mathrm{c}}+\boldsymbol{\Phi},
$$

где $\varphi_{\mathrm{c}}-$ нулевая мода, а $\boldsymbol{\Phi}$ обозначает ту часть поля, которая ортогональна нулевой моде:

$$
\int d^{2} x \boldsymbol{\Phi}(x)=0 .
$$

Вьполняя функциональное интегрирование по нулевой моде $\varphi_{c}$, получаем выражение

$$
\begin{aligned}
Z_{\Gamma}= & \left(\frac{\mu}{8 \pi}\right)^{s_{1}+s_{2}} \frac{1}{b^{2}|\operatorname{det} e|} \Gamma\left(-s_{1}\right) \Gamma\left(-s_{2}\right) \times \\
& \times \int \mathcal{D} \boldsymbol{\Phi} e^{-\int(\partial \boldsymbol{\Phi})^{2} /(8 \pi)+R_{g=0} \boldsymbol{\alpha}_{0} \Phi /(4 \pi)} \prod_{i=1}^{2 g+2} e^{\mathbf{d}_{i} \cdot \boldsymbol{\Phi}\left(x_{i}\right)} \times \\
& \times\left(\int d^{2} x e^{b \mathbf{e}_{1} \cdot \boldsymbol{\Phi}}\right)^{s_{1}}\left(\int d^{2} x e^{b \mathbf{e}_{2} \cdot \boldsymbol{\Phi}}\right)^{s_{2}} \int \mathcal{D} \tilde{\boldsymbol{\varphi}} e^{-\int(\partial \tilde{\boldsymbol{\varphi}})^{2} /(8 \pi)} \prod_{i=1}^{2 g+2} \sigma\left(x_{i}\right),
\end{aligned}
$$

где $s_{1}, s_{2}$ задаются как

$$
\begin{aligned}
& s_{1}=\left(b \operatorname{det} e_{i j}\right)^{-1}\left[-\alpha_{0} e_{22}+k_{1} e_{22}-k_{2} e_{21}\right], \\
& s_{2}=\left(b \operatorname{det} e_{i j}\right)^{-1}\left[-\alpha_{0} e_{12}+k_{2} e_{11}-k_{1} e_{12}\right],
\end{aligned}
$$

$\Gamma(x)$ - стандартная Г-функция и

$$
\mathbf{k}=\sum_{i=1}^{2 g+2} \mathbf{d}_{i} .
$$

Предполагая, что $s_{1}$ и $s_{2}$ - положительные целые, можно свести оставшийся функциональньй интеграл к функции распределения $W_{\Gamma}$-минимальной модели с помощью хорошо известных методов [14].

Благодарности. Работа одного из авторов (С.А. Апикяна) частично поддержана грантом INTAS-334. 


\section{Список литературы}

[1] E. Martinec. Quantum field theory: A guide for mathematicians. In: Theta functions. Proc. of 35th Summer Res. Inst. Bowdoin Coll., Brunswick/ME 1987, Proc. Symp. Pure Math. V. 49. Pt. 1. Eds. L. Ehrenpreis, R. C. Gunning. Providence, RI: AMS, 1989. P. 391.

[2] J. Polchinski. Superstring Theory. Cambridge: Camb. Univ. Press, 1998; M. B. Green, J.H. Schwarz, E. Witten. Superstring Theory. V. I, II. Cambridge: Camb. Univ. Press, 1987.

[3] M. A. Olshanetsky, A. M. Perelomov. Phys. Rep. 1981. V. 71. P. 313; И. М. Кричевер. УMH. 1977. T. 32. C. 185.

[4] N. Seiberg, E. Witten. Nucl. Phys. B. 1994. V. 426. P. 19; Erratum 1994. V. 430. P. 485.

[5] A. Marshakov. Seiberg-Witten Theory and Integrable Systems. Singapore: World Scientific, 1999.

[6] В. Г. Кац. Бесконечномерные алгебры Ли. М.: Мир, 1993; P. Goddard, D. Olive. Int. J. Mod. Phys. A. 1986. V. 1. P. 303.

[7] P. Bouwknegt, K. Schoutens. Phys. Rep. 1993. V. 223. P. 184.

[8] А. Б. Замолодчиков. ТМФ. 1985. Т. 65. С. 347; V. Fateev, A. B. Zamolodchikov. Nucl. Phys. B. 1987. V. 280. P. 644.

[9] A. М. Поляков. Лекция на семинаре в ИТФ им. Л. Д. Ландау 1982 (не опубл.); L. Takhtajan. Semiclasical Liouville theory, complex geometry of moduli space, and uniformization of Riemann surface. In: New Symmetry Principles in Quantum Field Theory. Eds. J. Frohlich et al. New York: Plenum Press, 1992.

[10] Al. B. Zamolodchikov. Nucl. Phys. B. 1987. V. 285. P. 481; V. Knizhnik. Commun. Math. Phys. 1987. V. 112. P. 567; L. Dixon, D. Friedan, E. Martinec, S. Shenker. Nucl. Phys. B. 1987. V. 282. P. 13; C. Crnkovic, G. Sotkov, M. Stanishkov. Phys. Lett. B. 1989. V. 220. P. 397; S. A. Apikyan, C. J. Efthimiou. Phys. Lett. B. 1995. V. 359 . P. 313.

[11] V.S. Dotsenko, V. A. Fateev. Nucl. Phys. B. 1984. V. 240. P. 312; 1985. V. 251. P. 691.

[12] С. А. Апикян, Ал. Б. Замолодчиков. ЖЭТФ. 1987. Т. 92. С. 34.

[13] A. Belavin, A. Polyakov, A. Zamolodchikov. Nucl. Phys. B. 1984. V. 241. P. 333.

[14] M. Goulian, M. Li. Phys. Rev. Lett. 1991. V. 66. P. 2051; A.B. Zamolodchikov, Al. B. Zamolodchikov. Nucl. Phys. B. 1996. V. 477. P. 577; H. Dorn, H. Otto. Phys. Lett. B. 1992. V. 291. P. 39; Nucl. Phys. B. 1994. V. 429. P. 375; А. Апикян, К. Эфтимиу. Письма в ЖЭТФ. 2001. Т. 74. С. 645; hep-th/0106272. 